OPEN ACCESS

Edited by:

Xiaoya Chen,

Shanghai Institute of Plant Physiology

and Ecology, China

Reviewed by:

Li Tian,

University of California, Davis,

United States

Tao Xia,

Anhui Agricultural University, China

${ }^{*}$ Correspondence:

Ying Wang

yingwang@scib.ac.cn

Specialty section:

This article was submitted to

Plant Metabolism

and Chemodiversity,

a section of the journal

Frontiers in Plant Science

Received: 26 May 2017

Accepted: 06 July 2017

Published: 19 July 2017

Citation:

Huang W, Lv H and Wang Y (2017)

Functional Characterization of a Novel

R2R3-MYB Transcription Factor Modulating the Flavonoid Biosynthetic Pathway from Epimedium sagittatum.

Front. Plant Sci. 8:1274.

doi: 10.3389/fpls.2017.01274

\section{Functional Characterization of a Novel R2R3-MYB Transcription Factor Modulating the Flavonoid Biosynthetic Pathway from Epimedium sagittatum}

\author{
Wenjun Huang ${ }^{1}$, Haiyan $L v^{1}$ and Ying Wang ${ }^{2,3 *}$ \\ ${ }^{1}$ Key Laboratory of Plant Germplasm Enhancement and Specialty Agriculture, Wuhan Botanical Garden, Chinese Academy \\ of Sciences, Wuhan, China, ${ }^{2}$ Key Laboratory of South China Agricultural Plant Molecular Analysis and Genetic \\ Improvement, South China Botanical Garden, Chinese Academy of Sciences, Guangzhou, China, ${ }^{3}$ Provincial Key \\ Laboratory of Applied Botany, South China Botanical Garden, Chinese Academy of Sciences, Guangzhou, China
}

Epimedium species have been widely used both as traditional Chinese medicinal plants and ornamental perennials. Both flavonols, acting as the major bioactive components (BCs) and anthocyanins, predominantly contributing to the color diversity of Epimedium flowers belong to different classes of flavonoids. It is well-acknowledged that flavonoid biosynthetic pathway is predominantly regulated by R2R3-MYB transcription factor (TF) as well as bHLH TF and WD40 protein at the transcriptional level. MYB TFs specifically regulating anthocyanin or flavonol biosynthetic pathway have been already isolated and functionally characterized from Epimedium sagittatum, but a R2R3-MYB TF involved in regulating both these two pathways has not been functionally characterized to date in Epimedium plants. In this study, we report the functional characterization of ESMYB9, a $R 2 R 3-M Y B$ TF previously isolated from $E$. sagittatum. The previous study indicated that EsMYB9 belongs to a small subfamily of R2R3-MYB TFs containing grape VVMYB5a and $V v M Y B 5 b$ TFs, which regulate flavonoid biosynthetic pathway. The present studies show that overexpression of ESMYB9 in tobacco leads to increased transcript levels of flavonoid pathway genes and increased contents of anthocyanins and flavonols. Yeast two-hybrid assay indicates that the C-terminal region of ESMYB9 contributes to the autoactivation activity, and ESMYB9 interacts with EsTT8 or AtTT8 bHLH regulator. Transient reporter assay shows that ESMYB9 slightly activates the expression of EsCHS (chalcone synthase) promoter in transiently transformed leaves of Nicotiana benthamiana, but the addition of AtTT8 or Es $T \pi 8 \mathrm{bHLH}$ regulator strongly enhances the transcriptional activation of EsMYB9 against five promoters of the flavonoid pathway genes except EsFLS (flavonol synthase). In addition, co-transformation of EsMYB9 and EsTT8 in transiently transfected tobacco leaves strongly induces the expressions of flavonoid biosynthetic genes. The potential role of EsMYB9 in modulating the biosynthesis and accumulation of sucrose-induced anthocyanin and flavonol-derived 
BCs is also discussed. These findings suggest that EsMYB9 is a novel R2R3-MYB $\mathrm{TF}$, which regulates the flavonoid biosynthetic pathway in Epimedium, but distinctly different with the anthocyanin or flavonol-specific MYB regulators identified previously in Epimedium plants.

Keywords: Epimedium, flavonoid, anthocyanin, flavonol, MYB, transcriptional regulation

\section{INTRODUCTION}

Herba Epimedii, an important traditional Chinese medicine, is derived from the dried aerial parts of Epimedium species in the family Berberidaceae (Huang et al., 2016a). In addition to traditionally being used as a kidney tonic and antirheumatic medicinal herb for more than 2000 years, Herba Epimedii has also been currently used for preventing or treating sexual dysfunction, osteoporosis, cardiovascular disease, tumor, and so on Ma et al. (2011) and Jiang et al. $(2015,2016)$. These significant therapeutic effects on human health are predominantly attributed to the flavonoid compounds, especially C8-prenylated flavonol glycosides, which have been well-confirmed to be the main bioactive components (BCs) in Epimedium plants (Ma et al., 2011; Jiang et al., 2015). Additionally, Epimedium species are also widely planted as groundcovers and ornamental plants in certain western countries, due to the attractive shape and the diverse color of foliage and flower (Zhang et al., 2014). A wide variety of flower color, ranging from white, yellow, pink, red to purple, is largely due to the accumulation of anthocyanins. Both anthocyanins and flavonolderived BCs belong to different classes of plant flavonoid metabolites.

As we know, the flavonoid biosynthetic pathway has been extensively and deeply studied in several model plant species, such as Arabidopsis, petunia, maize and grape, and almost all the structural genes of this pathway have been identified (Winkel-Shirley, 2001; Koes et al., 2005). In Epimedium plants, although the flavonoid-derived BCs have been phytochemically and pharmacologically well-studied previously (Ma et al., 2011; Jiang et al., 2015), the researches about their biosynthesis and regulation at the molecular level started late. In recent years, the flavonoid biosynthetic pathway in Epimedium sagittatum has been clarified, and majority of the structural genes of this pathway have been isolated (Zeng et al., 2013; Huang et al., 2015). Moreover, it has been well-acknowledged that the flavonoid biosynthetic pathway is predominantly controlled by MYB and bHLH transcription factors (TFs), together with WD40 protein at the transcriptional level (Hichri et al., 2011a; Xu et al., 2015). As an increasing number of MYB TFs involved in regulating flavonoid biosynthetic pathway have been identified from many plant species, and most of them are key regulators of the synthesis of anthocyanins (Liu et al., 2015). In Epimedium plants, three MYB regulators, including EsMYBA1, EsAN2 and $E S M Y B F 1$ have been also isolated and functionally characterized to regulate anthocyanin or flavonol biosynthetic pathway (Huang et al., 2013a, 2016a,b). However, there is another MYB gene, EsMYB9 previously isolated from E. sagittatum and suggested to probably regulate the flavonoid biosynthetic pathway (Huang et al., 2013b), but its function has not been clearly characterized until now.

It is well-documented that different R2R3-MYB family members specifically regulate the different branches of flavonoid pathway in both Arabidopsis and grape. For example, during the developing grape berries, $V v M Y B A 1$ and $V v M Y B A 2$ regulate anthocyanin synthesis (Kobayashi et al., 2004; Walker et al., 2007), VvMYBPA1 and VvMYBPA2 control proanthocyanidin (PA, also known as condensed tannin) synthesis (Bogs et al., 2007; Terrier et al., 2009), and $V v M Y B F 1$ specifically regulates flavonol synthesis (Czemmel et al., 2009). Similarly in Epimedium plants, EsMYBA1 and EsAN2 TFs regulated anthocyanin synthesis in a tissue-specific manner (Huang et al., 2013a, 2016b), while EsMYBF1 TF specifically regulated flavonol synthesis (Huang et al., 2016a). However, there is an existing small MYB clade, including $V v M Y B 5 a$ and $V v M Y B 5 b$ from grape, to regulate the whole flavonoid pathway, and their overexpression in tobacco can modulate the biosynthesis and accumulation of the different classes of flavonoids, including anthocyanin, PA, flavonol and lignin (Deluc et al., 2006, 2008). Corresponding in Epimedium plants, it was previously reported that EsMYB9 TF revealed a high level of sequence similarity with $V v M Y B 5 a$ and $V v M Y B 5 b$ regulators, and also phylogenetically related to the VvMYB5a/b clade (Huang et al., 2013b). Therefore, whether EsMYB9 has a similar function with $V v M Y B 5 a / b$ regulators to regulate the whole flavonoid pathway, especially anthocyanin and flavonol pathways is worthy of being further investigated.

Majority of $M Y B$ regulators of both anthocyanin and PA biosynthetic pathways generally depend on $b H L H$ TF as cofactor (Baudry et al., 2004; Gonzalez et al., 2008), while MYB regulator of flavonol biosynthetic pathway can function independently without bHLH cofactor (Mehrtens et al., 2005; Czemmel et al., 2009; Huang et al., 2016a). As for the VvMYB5a and $V v M Y B 5 b$ regulators of the flavonoid biosynthetic pathway, their abilities of interaction with $b H L H$ TF have not been described until now. Herein, the relationship analysis of EsMYB9 and $b H L H$ regulator was included in this study. In short, the putative EsMYB9 regulator of flavonoid biosynthetic pathway was functionally characterized in detail by overexpression in transgenic tobacco, yeast two-hybrid assay, dual-luciferase reporter assay and transient co-transformation in tobacco. The potential role of EsMYB9 in regulating the biosynthesis and accumulation of sucrose-induced anthocyanin and flavonolderived BCs in leaves of Epimedium was also discussed. Finally, the functional characterization of EsMYB9 regulator provides new insight into understanding the regulatory mechanisms of both anthocyanin and flavonol-derived BCs biosynthesis in Epimedium plants. 


\section{RESULTS}

\section{Overexpression of EsMYB9 in Tobacco Increases the Accumulation of Anthocyanins and Flavonols}

To characterize the role of ESMYB9 TF in regulating the flavonoid biosynthetic pathway, its overexpression in tobacco was carried out. The results of phenotypic changes showed that the red color of flower petals of the transgenic tobacco carrying EsMYB9 was remarkably enhanced, compared to that of control plants carrying the empty vector (Figures 1A,B). In particular, the MYB9-14 overexpression line had a strongest change of flower color. Moreover, the ends of filaments close to anther tissue from the overexpression lines turned from pale green to pink (Figure 1C). In addition, the whole filaments of flowers from the $T_{1}$ overexpression lines turned to pink (data not shown). This enhancement of red pigments was probably attributed to the increasing accumulation of anthocyanins. Therefore, the contents of flavonoids, including anthocyanins and flavonols in transgenic tobacco flowers were determined. The results indicated that the contents of total anthocyanin, anthocyanin cyanidin, flavonol (especially quercetin) were significantly increased in the MYB914 overexpression line, compared to the control (Figure 1D). As for other two overexpression lines (MYB9-4 and MYB9-17), only the flavonol quercetin content was moderately higher than that of control, but other flavonoids were not significantly increased (Figure 1D). Finally, these results suggested that overexpression of EsMYB9 in tobacco can lead to increase the accumulation of anthocyanins and flavonols in flowers.

\section{Overexpression of EsMYB9 in Tobacco Influences the Expression Levels of Flavonoid Pathway Genes}

The impact of EsMYB9 overexpression on the transcript levels of flavonoid pathway genes in transgenic tobacco flowers was analyzed by qPCR assay. The presence of introduced EsMYB9 in transgenic tobacco was firstly verified by semiquantitative RT-PCR assay. The results indicated that EsMYB9 was most abundantly expressed in the MYB9-14 overexpression line, followed by in the MYB9-4 overexpression line, and lowly expressed in the MYB9-17 overexpression line (Supplementary Figure S1). Secondly, the qPCR results showed that most of anthocyanin biosynthetic genes and two $b H L H$ regulatory genes, including $\mathrm{NtCHI}$ (chalcone isomerase), $\mathrm{NtF3} 3^{\prime} \mathrm{H}$ (flavonoid 3 '-hydroxylase), NtF3H (flavanone 3-hydroxylase), NtDFR (dihydroflavonol 4-reductase) and NtANS (anthocyanidin synthase) as well as NtAnla and $N t A n 1 b$ were significantly up-regulated in two overexpression lines (MYB9-4 and MYB9-14), especially in the MYB9-14 line, compared to the control (Figure 2). However, only in the MYB9-14 overexpression line, the expression level of NtFLS was slightly and significantly higher than that of the control (Figure 2). In addition, the transcript levels of almost all the general phenylpropanoid pathway genes, including NtPAL (phenylalanine ammonia-lyase), NtC4H (cinnamate 4-hydroxylase) and Nt4CL (4-coumaroyl:CoA-ligase) were not considerably changed by ectopic expression of EsMYB9, and they all were abundantly expressed in three overexpression lines and control plant (Figure 2). In the MYB9-17 overexpression lines carrying the lowest expression level of EsMYB9, the change extent of gene expression level was obviously lower than in other two overexpression lines (Figure 2). Finally, these results indicated that overexpression of EsMYB9 in tobacco strongly promoted the expression levels of main anthocyanin pathway genes and two $b H L H$ regulatory genes, and the effect on the NtFLS expression level have to be confirmed further.

\section{R2R3-MYB Regulator EsMYB9 Interacts with $b H L H$ Regulators}

Considering the presence of the conserved bHLH interacting motif in EsMYB9 protein and the up-regulation of NtAn1a and $N t A n 1 b$ bHLH regulators by ectopic expression of EsMYB9 in tobacco, yeast two-hybrid assay $(\mathrm{Y} 2 \mathrm{H})$ was carried out to detect the interaction of EsMYB9 with bHLH regulators. Autoactivation activity of EsMYB9 was firstly detected using three regions of EsMYB9 protein, including the coding region, the N-terminal and C-terminal region (Figure 3A). The results showed that the whole EsMYB9 protein possessed a strong activity of autoactivation, and the C-terminal revealed a stronger autoactivation activity but the N-terminal did not (Figure 3B), suggesting that the autoactivation activity of EsMYB9 TF must be attributed to the C-terminal region. For interaction analysis of EsMYB9 with bHLH proteins in $\mathrm{Y} 2 \mathrm{H}$ assay, three bHLH regulatory proteins of the flavonoid pathway, including EsTT8, EsGL3 from E. sagittatum and AtTT8 from Arabidopsis thaliana were selected. The results showed that the transformed yeast cells harboring EsMYB9-AD and EsTT8-BD, or EsGL3-BD or AtTT8-BD construct combinations were successfully passed through the double and quadruple selection plates, and the blue colonies occurred in the corresponding yeast cells through $\beta$-galactosidase assay (Figure 3C). Meanwhile, the yeast cells transformed with EsTT8-BD or AtTT8-BD plus with the empty $A D$ vector combination did not grow normally on the quadruple and the $\beta$-galactosidase selection plates, but the combination of EsGL3-BD and AD constructs passed through these selections (Figure 3C), indicating that EsGL3 possessed an activity of autoactivation, but EsTT8 and AtTT8 did not. In addition, both the positive and negative controls were as expected. Finally, the Y2H results indicated that EsMYB9 can interact with EsTT8 or AtTT8 bHLH regulators, but the interaction with EsGL3 can be excluded due to the presence of the autoactivation activity of EsGL3 protein found.

\section{$B H L H$ Is Required for Promoting the Transcriptional Activation of EsMYB9 against Promoters of the Flavonoid Pathway Genes}

Dual-luciferase reporter assay was performed in transiently transformed leaves of Nicotiana benthamiana to analyze the transcriptional activation of EsMYB9 TF against promoters of the flavonoid pathway genes from E. sagittatum with/without 

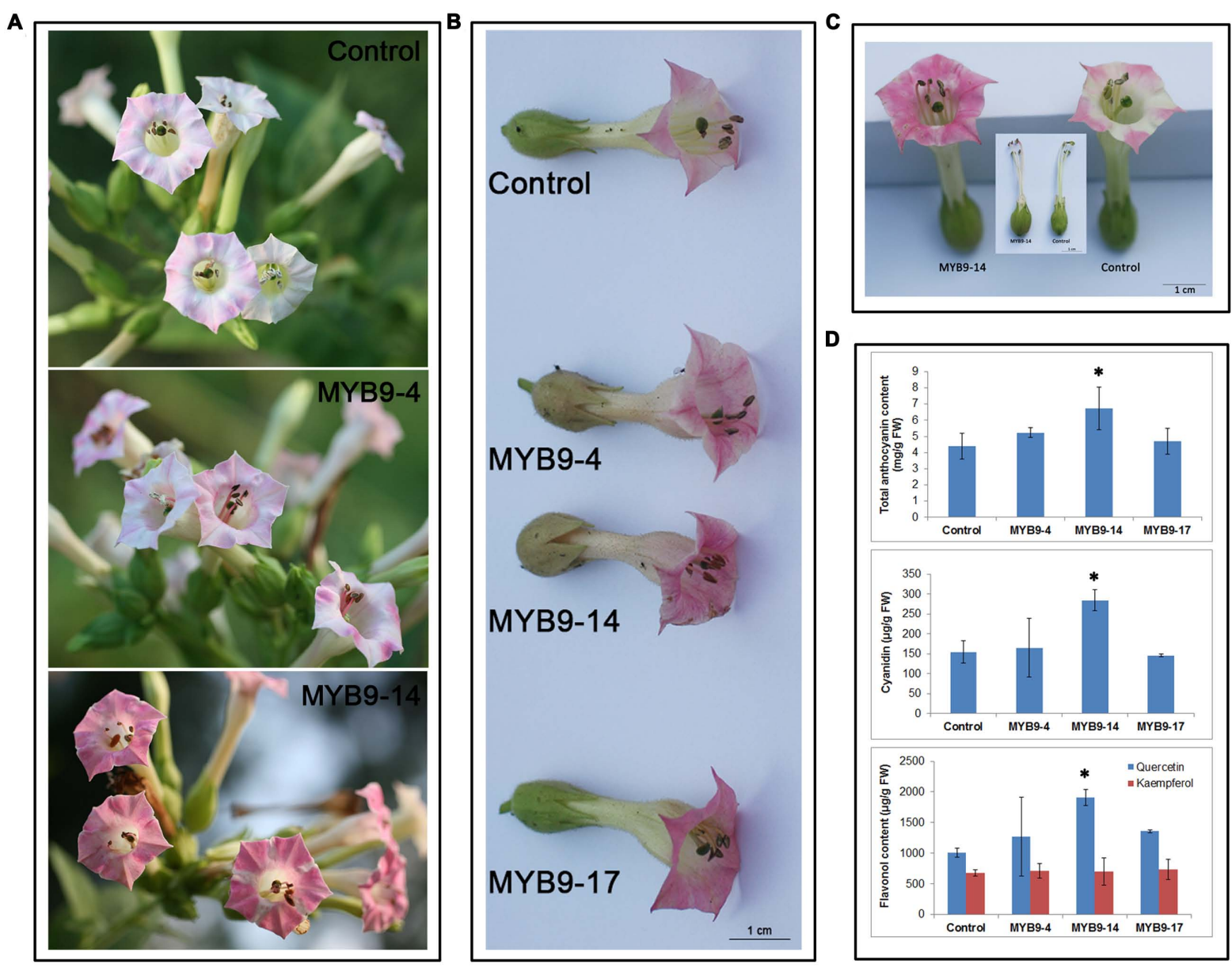

FIGURE 1 | Phenotype observation and flavonoid content measurement in transgenic tobacco overexpressing ESMYB9 gene. Three representative independent transgenic tobacco lines carrying ESMYB9 gene (MYB9-4, -14, and -17) and the transgenic tobacco carrying the empty vector as the control plant are indicated. Flowers on plants of the $T_{1}$ transgenic tobacco lines (A), flowers removed from the $T_{2}$ transgenic tobacco lines (B), comparison of flowers of the $T_{2}$ MYB9-14 line with the strongest change of phenotype and control plant (C), and flavonoid content (D), including total anthocyanin, cyanidin, and flavonol in transgenic tobacco flowers are showed. Each column represents mean values with SD bar from three biological replicates for each sample. Asterisk indicates a significant difference from the control plant $(P<0.05$, LSD test).

EsTT8 or AtTT8 bHLH regulator as a cofactor. The results showed that the LUC/REN ratios of promoters of EsCHS, $E s D F R 2$, and EsANS were increased by approximately 2 to 13-fold when EsMYB9 was used alone, but only EsCHS promoter activity was significantly enhanced, compared to their corresponding empty controls which only contained promoters without any MYB or bHLH TFs (Figure 4). In addition, the activities of EsDFR1, EsF3H and EsFLS promoters were not significantly changed by EsMYB9 used alone (Figure 4). It is noted that all the LUC/REN ratios of these six promoters treated with either the empty control or EsMYB9 alone were extremely low. However, the LUC/REN ratios of all these promoters except EsFLS were considerably increased when EsMYB9 with EsTT8 or AtTT8 bHLH regulators were co-transformed, while EsTT8 alone did not significantly promote the transcriptional activities of these promoters (Figure 4). Additionally, any treatment, including either EsMYB9 alone or its combination with $b H L H$ regulators did not largely change the transcriptional activity of EsFLS promoter, compared to the empty control (Figure 4). In summary, these results indicated that EsMYB9 was very likely to slightly activate the EsCHS promoter, but the addition of EsTT8 or AtTT8 bHLH TFs strongly promoted the transcriptional activation of EsMYB9 against promoters of the flavonoid pathway genes except EsFLS, suggesting that the $b H L H$ regulatory protein was required as a cofactor to enhance the transcription activation ability of EsMYB9 on target promoters in the heterologous system of N. benthamiana.

\section{Co-transformation of EsMYB9 and EsTT8 Strongly Induce the Expressions of Anthocyanin Biosynthetic Genes in Transiently Transformed Tobacco Leaves}

In order to further confirm the requirement of $b H L H$ regulator as cofactor for EsMYB9, the transient expression of EsMYB9 


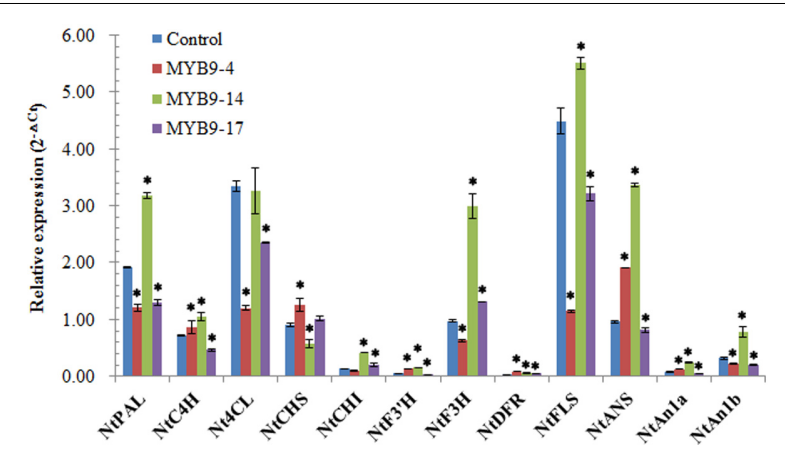

FIGURE 2 | Quantitative PCR assay of the flavonoid pathway genes in transgenic tobacco flowers carrying EsMYB9 gene. The expression levels of 10 structural genes of flavonoid biosynthetic pathway and two $\mathrm{bH} H \mathrm{H}$ regulatory genes (NtAn1a and NtAn1b) are determined by quantitative PCR assay. Tobacco Tub1 gene is used as an internal control, and comparative Ct method is used to determine the relative expression level. Three ESMYB9 overexpressing tobacco lines (MYB9-4, -14, and -17) and the control plant carrying the empty vector are used for qPCR assay. Each column represents average value with SD bar from three technical replicates. Asterisk represents a significant difference from the control plant at the level of $P<0.05$ using LSD statistical analysis.

with or without EsTT8 regulator was carried out in tobacco leaves. Firstly, the presence of EsMYB9 and EsTT8 in the corresponding transiently transformed tobacco leaves were confirmed by RT-PCR assay (Figure 5A). Secondly, quantitative PCR assay was performed to monitor the changes of the transcript levels of the flavonoid pathway genes. The results showed that both EsMYB9 and EsTT8 were abundantly expressed in tobacco leaves carrying itself alone or their combination (Figure 5B). Four structural genes, including $N t P A L, N t C 4 H$, $N t 4 C L$, and NtFLS were almost significantly down-regulated in all three treated samples, particularly in EsTT8 + EsMYB9 cotransformed sample, compared to the control sample transiently transformed by the empty vector (Figure 5C). The most striking change is that most of anthocyanin biosynthetic genes, including from $N t C H S$ to $N t U F 3 G T$ (UDP-glucose:flavonoid 3-O-glucosyltransferase) as well as NtAn1a and NtAn1b bHLH regulators were remarkably up-regulated in EsTT8+EsMYB9 cotransformed sample (Figure 5D). Meanwhile, these anthocyanin biosynthetic genes were not significantly changed by transient expression of either EsMYB9 or EsTT8 alone except NtUF3GT in EsMYB9 sample (Figure 5D). It is worthy to notice that the expression levels of the phenylpropanoid pathway gene, NtPAL, $N t C 4 H$, and $N t 4 C L$ were high in the four samples, but all the anthocyanin pathway genes were very lowly expressed in EsTT8 sample and EsMYB9 sample, closely to their background levels in the control sample (Figures 5C,D). However, surprisedly, no obvious red pigments occurred in transfected tobacco leaves carrying EsMYB9 or even with EsTT8 combination under an optical microscope (data not shown), implying that the anthocyanin accumulation may not be effectively induced by the transient expression of EsMYB9 with or without EsTT8 regulator. At least, it is well-confirmed that the cotransformation of EsMYB9 and EsTT8 can strongly induce the anthocyanin pathway genes in transiently transformed tobacco leaves.

\section{DISCUSSION}

It was previously reported that apart from the very wellconserved R2R3 MYB domain, three distinct conserved motifs were also found in EsMYB9 protein sequence, including the bHLH interaction motif, the $\mathrm{C} 1$ motif initially identified in the R2R3-MYB subgroup 4 proteins and the C3 motif specifically found in the all members of the VvMYB5a/b cluster (Huang et al., 2013b). Moreover, the high degree of sequence similarity and the close phylogenetic relationship of EsMYB9 with the members of the small VvMYB5a/b cluster may indicate similarity in function. First of all, overexpression of EsMYB9 in tobacco resulted in a similar phenotypic changes with the reports of $V v M Y B 5 a$ and $V v M Y B 5 b$ overexpression in tobacco. Pigmentation was clearly increased in petal and filament of transgenic tobacco flower overexpressing EsMYB9 (Figure 1), while flowers of transgenic tobacco overexpressing $V v M Y B 5 a$ or $V v M Y B 5 b$ showed a strongly increased pigmentation in both petal and stamen tissues (Deluc et al., 2006, 2008). However, it is noticed that the phenotypic changes were only observed in reproductive tissues, not in vegetative tissues of the EsMYB9 overexpression plants, which agrees with the reports of both $V v M Y B 5 a$ and $V v M Y B 5 b$ regulators. By contrast, overexpression of certain $M Y B$ regulators in tobacco induced a strongly increased pigmentation in both reproductive and vegetative tissues, such as EsMYBA1 and EsAN2 from E. sagittatum (Huang et al., 2013a, 2016b), AtPAP1 from A. thaliana (Borevitz et al., 2000) and NtAN2 from tobacco (Pattanaik et al., 2010), and all these $M Y B$ regulators have been identified to specifically regulate anthocyanin biosynthesis. Secondly, both anthocyanin and flavonol contents were significantly enhanced in transgenic flowers of the MYB9-14 overexpression line (Figure 1). It was previously reported that $V v M Y B 5 a$ overexpression increased the biosynthesis of anthocyanins, flavonols and condensed tannins and altered lignin metabolism in tobacco flowers (Deluc et al., 2006), while $V v M Y B 5 b$ overexpression resulted in a strong accumulation of anthocyanins and PAs in tobacco flowers (Deluc et al., 2008). These results indicated that the phenotypic change due to $E s M Y B 9$ overexpression was basically similar with that of $V v M Y B 5 a$ and $V v M Y B 5 b$, but appeared different with those $M Y B$ regulators of anthocyanin pathway.

The change of flavonoid content in transgenic tobacco flower implied that the expressions of flavonoid biosynthetic genes must be regulated by EsMYB9 overexpression. Corresponding to the increased anthocyanin in tobacco flowers expressing EsMYB9, most of anthocyanin pathway genes were remarkably up-regulated, including $N t C H I, N t F 3^{\prime} H, N t F 3 H, N t D F R$, and NtANS (Figure 2). Similarly, almost all the key anthocyanin structural genes, including $\mathrm{NtCHS}, \mathrm{NtCHI}, \mathrm{NtF} 3 \mathrm{H}, \mathrm{NtDFR}$, and NtANS were strongly up-regulated in the $V v M Y B 5 a$ or $V v M Y B 5 b$ overexpressing stamens and petals (Deluc et al., 2006, 2008). Additionally, the expression level of NtFLS was significantly decreased in two EsMYB9 overexpression lines and 


\section{A}
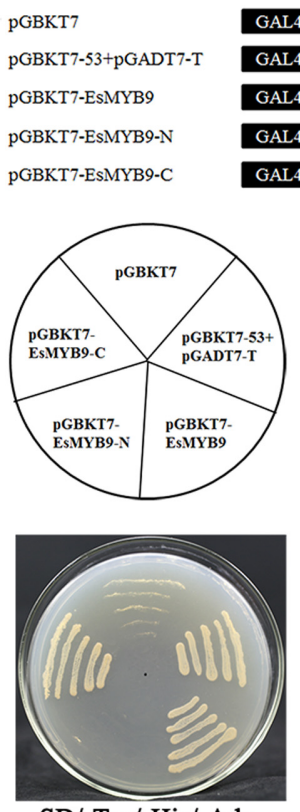

$\mathrm{SD} /$-Trp/-His/-Ade
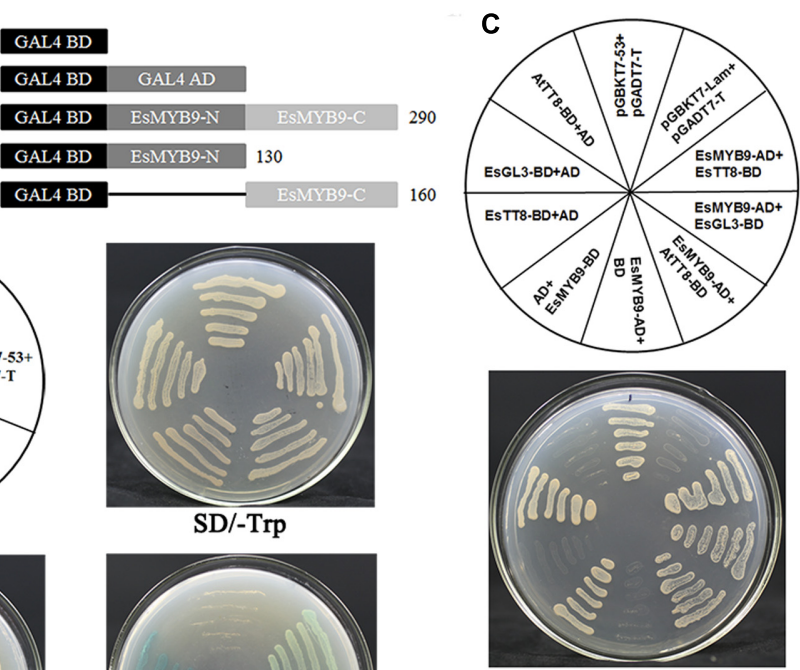

SD/-Leu/-Trp/-His/-Ade
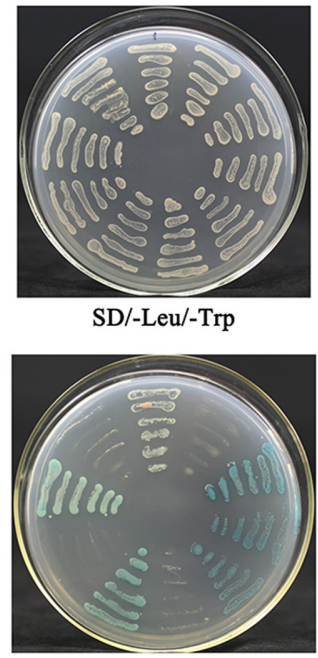

$\beta$-Galactosidase assay

FIGURE 3 | Yeast two-hybrid assay of EsMYB9 transcription factor (TF) with three bHLH regulators of the flavonoid pathway. Three different regions of EsMYB9 protein are used for transcription autoactivation assay, including the full-length coding region, the R2R3 MYB domain-containing N-terminal region and the C-terminal region (A). Transcription autoactivation assay (B) of EsMYB9 and its interaction with bHLH proteins (C) by yeast two-hybrid assay are indicated. Three bHLH proteins involved in regulation of the flavonoid biosynthetic pathway, EsTT8 and EsGL3 from Epimedium sagittatum and AtTT8 from Arabidopsis thaliana are used for yeast two-hybrid assay. Transformed yeast cells harboring pGBKT7-53 + pGADT7-T, or pGBKT7-Lam + pGADT7-T constructs are used as a positive and a negative control, respectively.

significantly increased only in the MYB9-14 line (Figure 2), which did not well-correspond to the increased flavonol in transgenic flowers. Although the flavonol content was slightly enhanced in transgenic tobacco flowers expressing $V v M Y B 5 a$, the transcript level of NtFLS was still not investigated (Deluc et al., 2006). For the general phenylpropanoid biosynthetic genes, including $N t P A L, N t C 4 H$ and Nt4CL, their expressions were not considerably changed in the EsMYB9 overexpression lines compared to the control, although both NtPAL and NtC4H were clearly increased in one of three EsMYB9 overexpression lines (Figure 2). Further in tobacco leaves transiently transformed by $E s M Y B$ 9, they were also not changed largely (Figure 5C). These results are partly consistent with the findings of VvMYB5a that the expressions of NtPAL and Nt4CL appeared unaffected, and $N t C 4 H$ was slightly induced by VvMYB5a overexpression in transgenic tobacco leaves (Deluc et al., 2006). Finally, these results indicated that overexpression of EsMYB9 in tobacco mainly promoted the expressions of anthocyanin biosynthetic genes.

Corresponding to the presence of bHLH interaction motif in EsMYB9 protein sequence, the interaction of EsMYB9 and EsTT8 or AtTT8 bHLH regulators was confirmed by $\mathrm{Y} 2 \mathrm{H}$ and dual-luciferase reporter assay (Figures 3, 4). To our knowledge, although $\mathrm{Y} 2 \mathrm{H}$ assay of $V v M Y B 5 a$ or $V v M Y B 5 b$ with $b H L H$ regulators have not been still investigated, but their interaction was demonstrated by a transient reporter assay (Deluc et al., 2008; Hichri et al., 2011b). As we know, majority of
$M Y B$ regulators of anthocyanin and PA biosynthetic pathways generally interact with $b H L H$ regulators as a cofactor to modulate the transcriptional expressions of flavonoid pathway genes, such as Epimedium EsMYBA1 and EsAN2 (Huang et al., 2013a, 2016b), and tobacco NtAN2 regulators (Pattanaik et al., 2010) of anthocyanin pathway, or Arabidopsis AtTT2 (Baudry et al., 2004), grape $V v M Y B P A 1, V v M Y B P A 2, V v M Y B P A R$ regulators (Bogs et al., 2007; Koyama et al., 2014) for PA pathway. Meanwhile, MYB regulators of flavonol pathway function independently without bHLH regulators, including AtMYB12 from A. thaliana (Mehrtens et al., 2005), VvMYBF1 from grape (Czemmel et al., 2009) and EsMYBF1 from E. sagittatum (Huang et al., 2016a). Moreover, the addition of EsTT8 or AtTT8 bHLH regulators strongly enhanced the transcription activation of EsMYB9 against the promoters of flavonoid biosynthetic genes except EsFLS promoter in a transient reporter assay, while EsMYB9 used alone just slightly activate the expression of EsCHS promoter (Figure 4). These results suggested that the presence of a bHLH protein was very critical, or even required for EsMYB9 to activate the expressions of target genes. The considerable up-regulation of the transcript levels of anthocyanin biosynthetic genes by co-transformation of EsMYB9 with EsTT8 in a transiently transformed tobacco leaves also support this conclusion (Figure 5). A similar result was also found for the activation assay of flavonoid gene promoters by $V v M Y B 5 a, V v M Y B 5 b, V v M Y B P A 1$, or $V v M Y B P A R$ regulators 
EsCHS Promoter

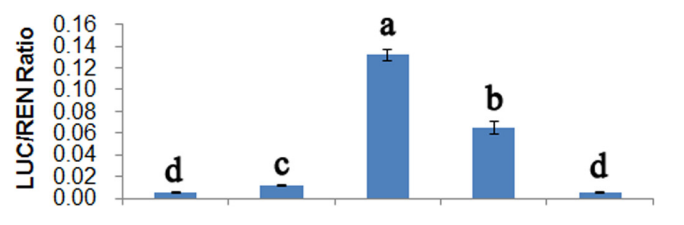

EsF3H Promoter

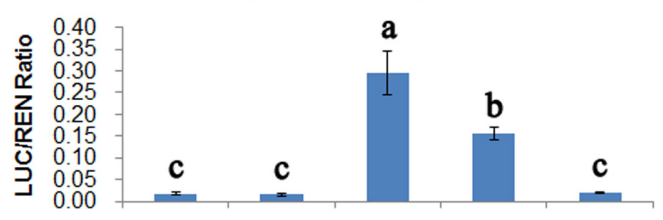

EsFLS Promoter

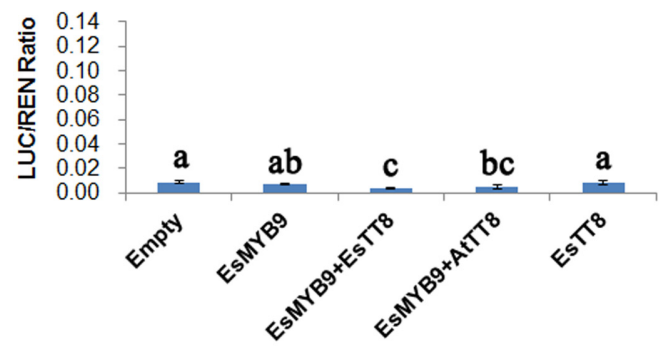

EsDFR1 Promoter

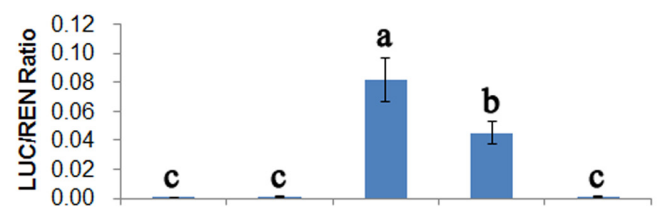

EsDFR2 Promoter

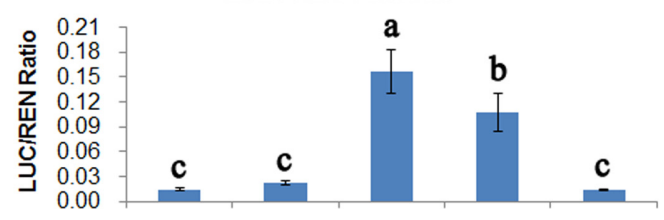

EsANS Promoter

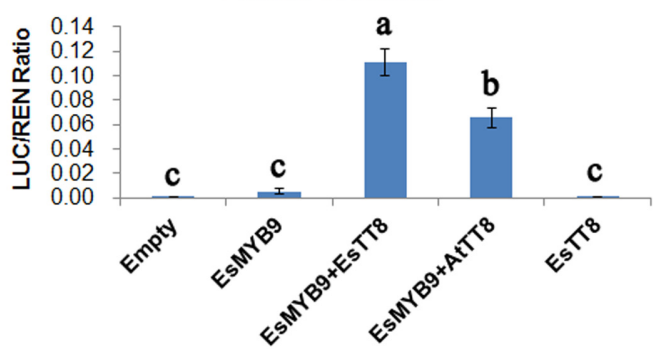

FIGURE 4 | Transcription activation assay of ESMYB9 against promoters of the flavonoid pathway genes with or without bHLH regulators in transiently transformed leaves of Nicotiana benthamiana. A total of six promoters of the flavonoid biosynthetic genes from E. sagittatum are used for dual-luciferase reporter assay, including ESCHS, ESDFR1, ESDFR2, ESF3H, ESFLS, and ESANS promoters. Two bHLH regulators of the flavonoid pathway, EsTT8 from E. sagittatum and AtTT8 from A. thaliana are also used as cofactors for EsMYB9. Transformed leaves carrying only the promoter-LUC reporter construct without the TF-containing effector construct are used as the controls (empty). The columns represent average values with SD bar from at least four biological replicates for each treatment. Values followed by the same letter are not significantly different at the level of $P<0.05$ (Duncan multiple range test).

in a transient reporter assay (Deluc et al., 2008; Hichri et al., 2011b; Koyama et al., 2014). They can't significantly and largely induce promoter activities without the expression of AtEGL3 or $V v M Y C 1$ bHLH regulator, and the addition of these $b H L H$ regulators resulted in a remarkably higher level of transcription activity of target promoter than that of these $M Y B$ regulators used alone. Surprisedly, the expression of EsFLS promoter appeared unaffected by expression of EsMYB9 with or without EsTT8 (Figure 4). This is probably because that an only $624 \mathrm{bp}$ upstream region from ATG start site of EsFLS used for the reporter construction is very short, and the potential binding site for EsMYB9 is absent in this promoter sequence. A longer promoter sequence of EsFLS may be needed to further detect its activity by EsMYB9 regulator.

A coordination relationship was previously suggested between anthocyanin pathway and flavonol-derived BCs pathway during the leaf developmental stage of E. sagittatum (Huang et al., 2015). As we know, sucrose treatment can modulate expressions of phenylpropanoid pathway genes and affect biosynthesis and accumulation of phenylpropanoids (Payyavula et al., 2013). For example in Arabidopsis, sucrose treatment can strongly induce accumulation of anthocyanins in seedlings through induction of AtPAP1 regulator (Teng et al., 2005). Therefore, the sterile cultured shoots of E. sagittatum were treated with 3 or $5 \%$ concentration of sucrose to dissect the potential role of EsMYB9 in modulating both anthocyanin and flavonol-derived BCs synthesis. The results showed that the contents of the main BCs, epimedin $\mathrm{C}$ and total flavonoid were significantly induced in response to sucrose treatment (Supplementary Figure S2A). In addition, red pigments were obviously observed in petioles and rhizomes 8 days after sucrose treatment. Corresponding, the expressions of flavonoid biosynthetic genes, including EsFLS, EsPT2, and EsPT3 for synthesis of the flavonol-derived BCs, and EsDFR1 and EsANS for anthocyanin biosynthesis were significantly up-regulated by sucrose treatment (Supplementary Figure S2B). Moreover, both EsMYBF1 and EsMYBA1 expressions were also significantly up-regulated, especially EsMYBF1, which are responsible for the regulation of flavonol pathway and anthocyanin pathway, respectively (Supplementary Figure S2C). However, EsMYB9 expression appeared unaffected by sucrose treatment, as well as EsAN2 and EsTT8 regulators (Supplementary Figure S2C). These results indicated that sucrose can induce both flavonol and anthocyanin synthesis and accumulation through inducing expressions of the biosynthetic genes of flavonol and anthocyanin pathways and their regulatory genes, mainly including EsMYBF1 and EsMYBA1, but not EsMYB9. Hence, EsMYB9 regulator appeared not involved in 


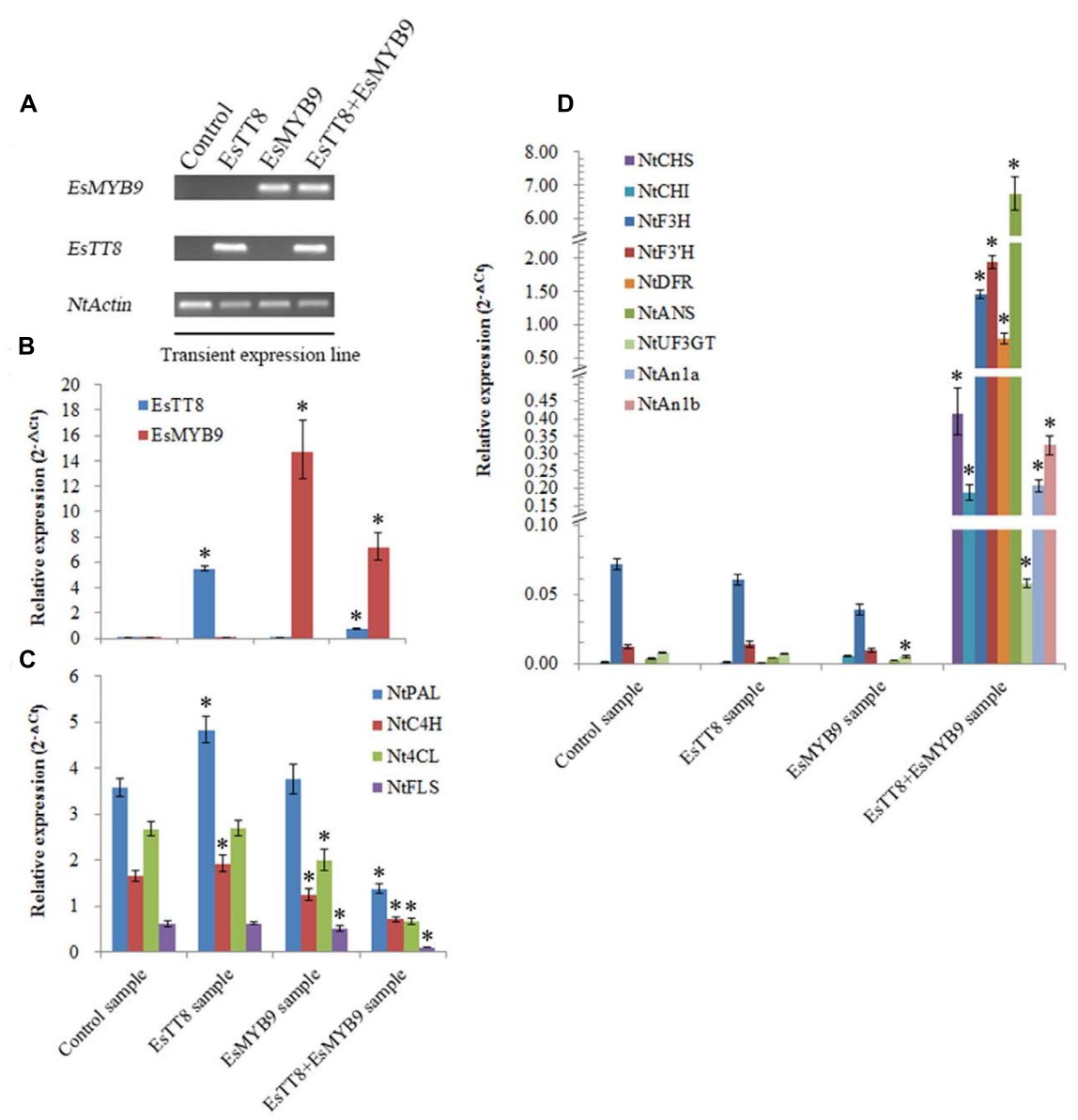

FIGURE 5 | Quantitative PCR assay of the flavonoid pathway genes in tobacco leaves transiently transfected by EsMYB9 with or without EsTT8 bHLH regulator. The leaves transiently transfected with the empty vector is used as the control sample, and transiently transfected with EsTT8 alone or EsMYB9 alone or their combination is referred to the EsTT8 sample, EsMYB9 sample and EsTT8+EsMYB9 sample, respectively. The presence of introduced EsMYB9 and EsTT8 genes in transiently transfected samples is confirmed by RT-PCR assay (A). The strong expressions of EsTT8 and EsMYB9 in transfected samples by their transient expressions (B), the down-regulation of four genes in transfected samples by EsTT8 or/and EsMYB9 transient expressions (C) and the remarkable up-regulation of nine genes in transiently co-transformed sample with EsTT8 and EsMYB9 (D) are indicated through quantitative PCR assay. Each column represents average value with SD bar from three technical replicates. Asterisk represents a significant difference from the control sample at the level of $P<0.05$ using LSD test.

the modulation of sucrose-induced anthocyanin and flavonol biosynthesis. In addition, the fact that EsTT8 bHLH regulator was not induced may partly contribute to the implementation of EsMYB9 function.

\section{CONCLUSION}

A novel R2R3-MYB TF, EsMYB9 isolated from E. sagittatum was previously suggested to regulate the flavonoid biosynthetic pathway. Here, this study indicates that overexpression of EsMYB9 in tobacco results in increased contents of both anthocyanin and flavonol in reproductive tissue through modulating the expressions of flavonoid pathway genes. EsMYB9 can interact with EsTT8 or AtTT8 bHLH regulators and these bHLH proteins are very critical for EsMYB9 transcription activation ability against target promoters. In addition, cotransformation of EsMYB9 and EsTT8 in transiently transfected tobacco leaves strongly induces the expressions of the main flavonoid biosynthetic genes except FLS gene. It is also demonstrated that EsMYB9 is not involved in the regulation of sucrose-induced anthocyanin and flavonol-derived BCs biosynthesis and accumulation in leaves of Epimedium. The functional characterization of $E s M Y B 9$ provides new insight into understanding the regulatory mechanism of the biosynthesis of both anthocyanin and flavonol-derived BCs in Epimedium plants.

\section{MATERIALS AND METHODS}

\section{Plant Materials}

Plants of Nicotiana tabacum cv. NC89 were used for stable and transient genetic transformation, and $N$. benthamiana was used for dual-luciferase reporter assay. Both they were grown in a greenhouse at $22-25^{\circ} \mathrm{C}$ with $14 \mathrm{~h} / 10 \mathrm{~h}$ of light/dark period until required. 


\section{Construction of Overexpression Vector and Tobacco Transformation}

For overexpression of EsMYB9 in tobacco, the coding region of EsMYB9 was transferred from pMD19-T cloning vector (Takara, Japan) cut with Sal I and Kpn I restriction enzymes to the modified binary pMV vector (derived from pBI121 vector) cut with Xho I and Kpn I restriction enzymes, producing the pMV-EsMYB9 overexpression construct. The EsMYB9 expression was triggered under the control of CaMV $35 \mathrm{~S}$ promoter in this construct. This overexpression construct was confirmed by sequencing and then introduced into Agrobacterium tumefaciens EHA105 strain by electroporation method for tobacco stable genetic transformation. The leaf disk method described previously (Horsch et al., 1985) was used for Agrobacterium-mediated genetic transformation of tobacco. The positive transformed tobacco plants were screened using kanamycin as a plant selective marker and the existence of introduced EsMYB9 gene in transformants was confirmed by PCR assay. Finally, three representative independent $T_{2}$ transgenic tobacco lines (MYB9-4, -14, and -17) showing the strong, moderate and weak changes of flower colors were used for further analysis. In addition, the transient expressions of EsMYB9 or EsTT8 or their combination in tobacco leaves were also carried out as described for the Agrobacterium-infiltrated transformation of N. benthamiana (Huang et al., 2016a). The detailed protocol was seen in the method of dual-luciferase reporter assay below. The overexpression construct pMV-EsTT8 harboring the full-length coding region of EsTT8 was used for the transient expression. For the transient co-transformation of EsMYB9 and EsTT8, their suspension culture were equally mixed as the infiltration solution. The infiltrated pots of leaves were harvested for qPCR assay after 3 days of inoculation. Transgenic tobacco expressing the pMV empty vector was used as a negative control in either stable transformation or transient transformation. Primers for the overexpression vector constructions of EsMYB9 and EsTT8 were listed in Supplementary Table S1.

\section{Measurement of Flavonoid Content in Transgenic Tobacco Flowers}

To clarify the effect of EsMYB9 overexpression on flavonoid synthesis and accumulation in transgenic tobacco flowers, the flavonoid extraction and content measurement were performed as described previously (Huang et al., 2015). Total anthocyanin content and flavonoid content were measured using a spectrophotometric method and a HPLC (high performance liquid chromatography) method, respectively. Briefly, the buffer of $1 \% \mathrm{HCl} /$ methanol was used to extract anthocyanin from tobacco flowers and the absorbance of supernatant was monitored at 530 and $657 \mathrm{~nm}$. The formula A530-0.25*A657 was adopted to compensate for the absorption of chlorophyll degradation products at $530 \mathrm{~nm}$. Total anthocyanin content was determined using a cyanidin-3-O-glucoside as a standard. Meanwhile, flavonols were extracted by $80 \%$ methanol and sonication. Both anthocyanin and flavonol contents were presented as aglycones by preparing acid-hydrolyzed extracts. Before injection, a $0.22 \mu \mathrm{m}$ filter membrane was used to filter the hydrolyzation solution. The chromatographic analysis was run on an Agilent 1100 series HPLC system accompanied with an Agilent TC-C18 column $(5 \mu \mathrm{m}, 4.6 \mathrm{~mm} \times 250 \mathrm{~mm})$. The mobile phase was comprised of solvent A $(0.1 \%$ formic acid in water), solvent B (acetonitrile) and solvent C (methanol). The gradient elution program was as follows: initiate from $10 \% \mathrm{~B}+2 \% \mathrm{C}$ at $0 \mathrm{~min}$; increase to $20 \% \mathrm{~B}+4 \% \mathrm{C}$ at $10 \mathrm{~min}$; proceed up to $50 \% \mathrm{~B}+10 \% \mathrm{C}$ at $15 \mathrm{~min}$; drop to $20 \% \mathrm{~B}+4 \% \mathrm{C}$ at $20 \mathrm{~min}$; back to the initial at $25 \mathrm{~min}$ and re-equilibrate for $3 \mathrm{~min}$. The detection wavelength for kaempferol and quercetin flavonols, and cyanidin anthocyanin was set at 350 and $530 \mathrm{~nm}$, respectively. The identification and quantification of flavonoid compounds in tobacco flowers were compared with the commercial standards of kaempferol and quercetin, and cyanidin. The measurement of flavonoid content for each sample was repeated two times using three independent biological replicates.

\section{Quantitative RT-PCR Assay}

qRT-PCR assay was carried out to determine the gene expression level according to Huang et al. (2013a). In brief, total RNA was isolated from flowers and leaves of tobacco using RNAiso plus reagent (Takara, Japan) and its quality and quantity were determined by electrophoresis and NanoDrop 2000 spectrophotometer (Thermo Scientific, United States). Before reverse transcription, gDNA eraser (Takara, Japan) was used to digest total RNA for removing any contaminated genomic DNA. Then, the digested RNA solution was reversely transcribed by PrimeScript RT reagent kit (Takara, Japan) following the supplier's recommendation. Finally, the qPCR assay was set up with SYBR Premix Ex Taq II (Tli RNase H Plus) kit (Takara, Japan) and performed in an ABI7500 Fast Real-Time PCR equipment (Applied Biosystems, United States) following the manufacturer's instruction. The qPCR program was as follows: $95^{\circ} \mathrm{C}$ for $30 \mathrm{~s}, 40$ cycles of $95^{\circ} \mathrm{C}$ for $3 \mathrm{~s}$ and $60^{\circ} \mathrm{C}$ for $30 \mathrm{~s}$, and a default melt curve program. The comparative $\mathrm{Ct}$ method was used to analyze the gene expression level (Schmittgen and Livak, 2008). Tobacco NtTub1 genes was used as an internal control of qPCR assay. Primers used for qPCR assay of the flavonoid pathway genes in tobacco were listed in Supplementary Table S2.

\section{Yeast Two-Hybrid Assay of EsMYB9 and bHLH TFs}

To analyze the interaction of EsMYB9 with bHLH TFs known to regulate the flavonoid biosynthetic pathway, Y2H assay was performed as previously described by Huang et al. (2016a). The pGADT7 and pGBKT7 vectors (Clontech, Japan) harboring the GAL4 activation domain (AD) and GAL4 DNA-binding domain (BD), respectively, were used for $\mathrm{Y} 2 \mathrm{H}$ assay. Firstly, three different regions of EsMYB9 protein, the coding region, MYB domain (aa 1-130) and C-terminal region (aa 131-290) (Figure 3A), were inserted into the pGBKT7 vector, generating the EsMYB9-BD, EsMYB9-N-BD 
and EsMYB9-C-BD constructs for autoactivation activity assay, respectively. For $\mathrm{Y} 2 \mathrm{H}$ assay, the full-length coding region of EsMYB9 was subcloned into the pGADT7 vector, generating the EsMYB9-AD construct, and the EsGL3-BD construct was generated by the full-length coding region of EsGL3 bHLH TF (GenBank Accession: KJ010529) inserted into the pGBKT7 vector. All primers used for $\mathrm{Y} 2 \mathrm{H}$ constructs were listed in Supplementary Table S1. In addition, the AtTT8BD and EsTT8-BD constructs which contain the AtTT8 or EsTT8 bHLH protein fused with BD domain had been described previously (Huang et al., 2016b). These plasmid constructs were transformed into yeast strain AH109 using the LiAc/SS carrier DNA/PEG method (Gietz and Schiestl, 2007). Transformed yeast cells were consecutively plated on the double (SD/-Trp/-Leu), quadruple (SD/-Trp/-Leu/Ade/-His) dropout medium, and quadruple dropout medium supplemented with X-Gal substrate for $\beta$-galactosidase assay. The growth of transformed yeast cells was photographed 3-4 days after incubation. Transformed yeast cells harboring pGBKT7$53+$ pGADT7-T, pGBKT7-Lam + pGADT7-T construct combinations were selected as a positive and a negative control, respectively.

\section{Dual-Luciferase Reporter Assay of Transiently Transformed Leaves of Nicotiana benthamiana}

Transcription activation of EsMYB9 against promoters of the flavonoid pathway genes from E. sagittatum was performed using a dual-luciferase reporter assay in the transiently transformed leaves of $N$. benthamiana as previously described (Huang et al., 2016a). The full-length coding region of EsMYB9 was transferred from pMD19-T vector into the pGreen II 62-SK vector (Hellens et al., 2005), generating the EsMYB9containing effector construct. Primers for EsMYB9 effector construction were listed in Supplementary Table S1. In addition, a total of six reporter constructs containing EsCHS, EsDFR1, EsDFR2, EsF3H, EsFLS, or EsANS promoters in the pGreen II 0800-LUC reporter vector, and two effector constructs containing EsTT8 or AtTT8 bHLH TFs in the pGreen II $62-S K$ vector were as previously described by Huang et al. (2016a).

These reporter and effector constructs were introduced into Agrobacterium tumefaciens GV3101 strain using a electroporation method. Transformed Agrobacterium cells were grown on LB agar supplemented with rifampicin, gentamycin, and kanamycin antibiotics and incubated at $28^{\circ} \mathrm{C}$ for $2-3$ days. The confluent bacteria was scraped and suspended in the infiltration buffer $[10 \mathrm{mM}$ MES ( $\mathrm{pH} 5.5)$, $10 \mathrm{mM} \mathrm{MgCl}_{2}, 150 \mu \mathrm{M}$ acetosyringone] to an $\mathrm{OD}_{600}$ of $0.2-0.3$, and then was kept at room temperature without shaking for at least $2-3 \mathrm{~h}$ before infiltration. An aliquot of $100 \mu \mathrm{L}$ of Agrobacterium culture transformed with the promotercontaining reporter construct and an aliquot of $450 \mu \mathrm{L}$ of a second Agrobacterium cultures transformed with the MYB and/or bHLH-containing effector constructs were mixed for transient transformation. Approximately $200 \mu \mathrm{L}$ of this culture mixture was infiltrated into three-four young leaves of each plant, with two-three points for each leaf. At least four-five independent plants were used for each treatment. Finally, the dual-luciferase assay was carried out 3-4 days after inoculation.

A Dual-Luciferase Reporter Assay System kit (Promega, United States) was used to perform the dual-luciferase assay of transiently transformed leaves of $N$. benthamiana, following the method described previously by Huang et al. (2016a). Briefly speaking, the leaf disks with a diameter of $1 \mathrm{~cm}$ were collected from the infiltrated points using a puncher, and finely ground in $500 \mu \mathrm{L}$ of Passive Lysis Buffer. This crude extract was diluted 50 -fold with Passive Lysis Buffer and then an aliquot of $10 \mu \mathrm{L}$ of diluent was taken out and measured in $40 \mu \mathrm{L}$ of Luciferase Assay Buffer. Another $40 \mu \mathrm{L}$ of Stop and Glow Buffer was then added and a second luminescence measurement was recorded. The measurement of luminescence unit was carried out using a GloMax 20/20 luminometer (Promega, United States) with a $5 \mathrm{~s}$ delay and $10 \mathrm{~s}$ integrated measurement. Transcriptional activity data were presented as the ratio of LUC to REN luminescent activity. Only the promoter-LUC reporter constructs (without any TF) were used as the background controls.

\section{AUTHOR CONTRIBUTIONS}

WH and YW initiated and designed the research. WH performed and analyzed the experiments. HL contributed to materials. WH wrote the paper, and YW revised the paper.

\section{ACKNOWLEDGMENT}

This study was supported by the fund of National Natural Science Foundation of China (31270340) and Scientific Project of Ningxia Agriculture Comprehensive Development (znnfkj2015-01).

\section{SUPPLEMENTARY MATERIAL}

The Supplementary Material for this article can be found online at: http://journal.frontiersin.org/article/10.3389/fpls.2017.01274/ full\#supplementary-material

FIGURE S1 | Gel image of semi-quantitative RT-PCR assay of EsMYB9 in transgenic tobacco flowers carrying EsMYB9 gene. Three MYB9 expressing transgenic tobacco lines and the control plant carrying the empty vector were used for semi-quantitative RT-PCR assay, and tobacco Actin gene was used as a reference.

FIGURE S2 | Flavonoid content measurement and qPCR assay of the flavonoid-related genes in sucrose-treated leaves of GD1 population of Epimedium sagittatum. Plant of GD1 population that contain only epimedin C as the main bioactive components was used as materials for sucrose treatment. The sterilized shoots were cultured in MS basal medium supplemented with 3 or $5 \%$ (W/v) of sucrose. The young leaves and petioles were harvested 8 days after treatment for further analysis. The content of epimedin $\mathrm{C}$ and total flavonoid (A), the expression levels of flavonoid biosynthetic genes $\mathbf{( B )}$ and regulatory genes (C) by qPCR assay were indicated. Each column represents mean value with SD bar from three replicates. Asterisk indicates a significant difference from the sucrose $3 \%$ used as the control $(P<0.05$, LSD test). 


\section{REFERENCES}

Baudry, A., Heim, M., Dubreucq, B., Caboche, M., Weisshaar, B., and Lepiniec, L. (2004). TT2, TT8, and TTG1 synergistically specify the expression of BANYULS and proanthocyanidin biosynthesis in Arabidopsis thaliana. Plant J. 39, 366-380. doi: 10.1111/j.1365-313X.2004.02138.x

Bogs, J., Jaffé, F. W., Takos, A. M., Walker, A. R., and Robinson, S. P. (2007). The grapevine transcription factor VvMYBPA1 regulates proanthocyanidin synthesis during fruit development. Plant Physiol. 143, 1347-1361. doi: 10.1104/ pp.106.093203

Borevitz, J. O., Xia, Y., Blount, J., Dixon, R. A., and Lamb, C. (2000). Activation tagging identifies a conserved MYB regulator of phenylpropanoid biosynthesis. Plant Cell 12, 2383-2393. doi: 10.1105/tpc.12.12.2383

Czemmel, S., Stracke, R., Weisshaar, B., Cordon, N., Harris, N. N., Walker, A. R., et al. (2009). The grapevine R2R3-MYB transcription factor VvMYBF1 regulates flavonol synthesis in developing grape berries. Plant Physiol. 151, 1513-1530. doi: 10.1104/pp.109.142059

Deluc, L., Barrieu, F., Marchive, C., Lauvergeat, V., Decendit, A., Richard, T., et al. (2006). Characterization of a grapevine R2R3-MYB transcription factor that regulates the phenylpropanoid pathway. Plant Physiol. 140, 499-511. doi: 10.1104/pp.105.067231

Deluc, L., Bogs, J., Walker, A. R., Ferrier, T., Decendit, A., Merillon, J.-M., et al. (2008). The transcription factor VvMYB5b contributes to the regulation of anthocyanin and proanthocyanidin biosynthesis in developing grape berries. Plant Physiol. 147, 2041-2053. doi: 10.1104/pp.108.118919

Gietz, R. D., and Schiestl, R. H. (2007). High-efficiency yeast transformation using the LiAc/SS carrier DNA/PEG method. Nat. Protoc. 2, 31-34. doi: 10.1038/ nprot.2007.13

Gonzalez, A., Zhao, M., Leavitt, J. M., and Lloyd, A. M. (2008). Regulation of the anthocyanin biosynthetic pathway by the TTG1/bHLH/Myb transcriptional complex in Arabidopsis seedlings. Plant J. 53, 814-827. doi: 10.1111/j.1365313X.2007.03373.x

Hellens, R. P., Allan, A. C., Friel, E. N., Bolitho, K., Grafton, K., Templeton, M. D., et al. (2005). Transient expression vectors for functional genomics, quantification of promoter activity and RNA silencing in plants. Plant Methods 1, 13-26. doi: 10.1186/1746-4811-1-13

Hichri, I., Barrieu, F., Bogs, J., Kappel, C., Delrot, S., and Lauvergeat, V. (2011a). Recent advances in the transcriptional regulation of the flavonoid biosynthetic pathway. J. Exp. Bot. 62, 2465-2483. doi: 10.1093/jxb/erq442

Hichri, I., Deluc, L., Barrieu, F., Bogs, J., Mahjoub, A., Regad, F., et al. (2011b). A single amino acid change within the R2 domain of the VvMYB5b transcription factor modulates affinity for protein partners and target promoters selectivity. BMC Plant Biol. 11:117. doi: 10.1186/1471-2229-11-117

Horsch, R., Fry, J., Hoffmann, N., Eichholtz, D., Rogers, S. G., and Fraley, R. (1985). A simple and general method for transferring genes into plants. Science 227, 1229-1231. doi: 10.1126/science.227.4691.1229

Huang, W., Khaldun, A. B. M., Chen, J., Zhang, C., Lv, H., Yuan, L., et al. (2016a). A R2R3-MYB transcription factor regulates the flavonol biosynthetic pathway in a traditional Chinese medicinal plant, Epimedium sagittatum. Front. Plant Sci. 7:1089. doi: 10.3389/fpls.2016.01089

Huang, W., Khaldun, A. B. M., Lv, H., Du, L., Zhang, C., and Wang, Y. (2016b). Isolation and functional characterization of a R2R3-MYB regulator of the anthocyanin biosynthetic pathway from Epimedium sagittatum. Plant Cell Rep. 35, 883-894. doi: 10.1007/s00299-015-1929-z

Huang, W., Sun, W., Lv, H., Luo, M., Zeng, S., Pattanaik, S., et al. (2013a). A R2R3MYB transcription factor from Epimedium sagittatum regulates the flavonoid biosynthetic pathway. PLoS ONE 8:e70778. doi: 10.1371/journal.pone.0070778

Huang, W., Sun, W., Lv, H., Xiao, G., Zeng, S., and Wang, Y. (2013b). Isolation and molecular characterization of thirteen R2R3-MYB transcription factors from Epimedium sagittatum. Int. J. Mol. Sci. 14, 594-610. doi: 10.3390/ijms14 010594

Huang, W., Zeng, S., Xiao, G., Wei, G., Liao, S., Chen, J., et al. (2015). Elucidating the biosynthetic and regulatory mechanisms of flavonoid-derived bioactive components in Epimedium sagittatum. Front. Plant Sci. 6:689. doi: 10.3389/fpls. 2015.00689

Jiang, J., Song, J., and Jia, X.-B. (2015). Phytochemistry and ethnopharmacology of Epimedium L. species. Chin. Herb. Med. 7, 204-222. doi: 10.1016/S16746384(15)60043-0
Jiang, J., Zhao, B.-J., Song, J., and Jia, X.-B. (2016). Pharmacology and clinical application of plants in Epimedium L. Chin. Herb. Med. 8, 12-23. doi: 10.1016/ S1674-6384(16)60003-5

Kobayashi, S., Goto-Yamamoto, N., and Hirochika, H. (2004). Retrotransposoninduced mutations in grape skin color. Science 304, 982. doi: 10.1126/science. 1095011

Koes, R., Quattrocchio, F., and Mol, J. (2005). The flavonoid biosynthetic pathway in plants: function and evolution. BioEssays 16, 123-132. doi: 10.1002/bies. 950160209

Koyama, K., Numata, M., Nakajima, I., Goto-Yamamoto, N., Matsumura, H., and Tanaka, N. (2014). Functional characterization of a new grapevine MYB transcription factor and regulation of proanthocyanidin biosynthesis in grapes. J. Exp. Bot. 65, 4433-4449. doi: 10.1093/jxb/eru213

Liu, J., Osbourn, A., and Ma, P. (2015). MYB transcription factors as regulators of phenylpropanoid metabolism in plants. Mol. Plant 8, 689-708. doi: 10.1016/j. molp.2015.03.012

Ma, H., He, X., Yang, Y., Li, M., Hao, D., and Jia, Z. (2011). The genus Epimedium: an ethnopharmacological and phytochemical review. J. Ethnopharmacol. 134, 519-541. doi: 10.1016/j.jep.2011.01.001

Mehrtens, F., Kranz, H., Bednarek, P., and Weisshaar, B. (2005). The Arabidopsis transcription factor MYB12 is a flavonol-specific regulator of phenylpropanoid biosynthesis. Plant Physiol. 138, 1083-1096. doi: 10.1104/pp.104.058032

Pattanaik, S., Kong, Q., Zaitlin, D., Werkman, J. R., Xie, C. H., Patra, B., et al. (2010). Isolation and functional characterization of a floral tissue-specific R2R3 MYB regulator from tobacco. Planta 231, 1061-1076. doi: 10.1007/s00425-010$1108-\mathrm{y}$

Payyavula, R. S., Singh, R. K., and Navarre, D. A. (2013). Transcription factors, sucrose, and sucrose metabolic genes interact to regulate potato phenylpropanoid metabolism. J. Exp. Bot. 64, 5115-5131. doi: 10.1093/jxb/ ert303

Schmittgen, T. D., and Livak, K. J. (2008). Analyzing real-time PCR data by the comparative CT method. Nat. Protoc. 3, 1101-1108. doi: 10.1038/nprot.2008.73

Teng, S., Keurentjes, J., Bentsink, L., Koornneef, M., and Smeekens, S. (2005). Sucrose-specific induction of anthocyanin biosynthesis in Arabidopsis requires the MYB75/PAP1 gene. Plant Physiol. 139, 1840-1852. doi: 10.1104/pp.105. 066688

Terrier, N., Torregrosa, L., Ageorges, A., Vialet, S., Verries, C., Cheynier, V., et al. (2009). Ectopic expression of VvMybPA2 promotes proanthocyanidin biosynthesis in grapevine and suggests additional targets in the pathway. Plant Physiol. 149, 1028-1041. doi: 10.1104/pp.108.131862

Walker, A. R., Lee, E., Bogs, J., McDavid, D. A. J., Thomas, M. R., and Robinson, S. P. (2007). White grapes arose through the mutation of two similar and adjacent regulatory genes. Plant J. 49, 772-785. doi: 10.1111/j.1365-313X.2006. 02997.x

Winkel-Shirley, B. (2001). Flavonoid biosynthesis. a colorful model for genetics, biochemistry, cell biology, and biotechnology. Plant Physiol. 126, 485-493. doi: $10.1104 / p p .126 .2 .485$

Xu, W., Dubos, C., and Lepiniec, L. (2015). Transcriptional control of flavonoid biosynthesis by MYB-bHLH-WDR complexes. Trends Plant Sci. 20, 176-185. doi: 10.1016/j.tplants.2014.12.001

Zeng, S., Liu, Y., Hu, W., Liu, Y., Shen, X., and Wang, Y. (2013). Integrated transcriptional and phytochemical analyses of the flavonoid biosynthesis pathway in Epimedium. Plant Cell Tissue Organ Cult. 115, 355-365. doi: 10. 1007/s11240-013-0367-2

Zhang, Y., Yang, L., Chen, J., Sun, W., and Wang, Y. (2014). Taxonomic and phylogenetic analysis of Epimedium L. based on amplified fragment length polymorphisms. Sci. Hortic. 170, 284-292. doi: 10.1016/j.scienta.2014.02.025

Conflict of Interest Statement: The authors declare that the research was conducted in the absence of any commercial or financial relationships that could be construed as a potential conflict of interest.

Copyright (c) 2017 Huang, Lv and Wang. This is an open-access article distributed under the terms of the Creative Commons Attribution License (CC BY). The use, distribution or reproduction in other forums is permitted, provided the original author(s) or licensor are credited and that the original publication in this journal is cited, in accordance with accepted academic practice. No use, distribution or reproduction is permitted which does not comply with these terms. 